\title{
Design of Automatic Thruster Assisted Position Mooring Systems for Ships
}

\author{
JANN PETER STRAND $\dagger$, ASGEIR J. SØRENSEN* and \\ THOR I. FOSSEN $\dagger$
}

Keywords: Position mooring, model-based control, hydrodynamics, marine systems

This paper addresses the mathematical modelling and controller design of an automatic thruster assisted position mooring system. Such control systems are applied to anchored floating production offloading and storage vessels and semi-subs. The controller is designed using model based control with a LQG feedback controller in conjunction with a Kalman filter. The controller design is in addition to the environmental loads accounting for the mooring forces acting on the vessel. This is reflected in the model structure and in the inclusion of new functionality.

\section{Introduction}

A position mooring (PM) system is a control system for automatic thruster assistance of moored structures, Fig. 1. Such systems are important for safe operation of anchored floating production storage and off-loading vessels (FPSO's) and semi-submersibles. For the oil companies the FPSO concept has proved to be a cost efficient and flexible solution to extract oil, especially from marginal fields.

In position mooring the thruster assistance will be complementary to the mooring system. Normally, most of the station keeping in surge and sway will be provided by the mooring system. This is in contrast to dynamic positioning (DP) of non-anchored

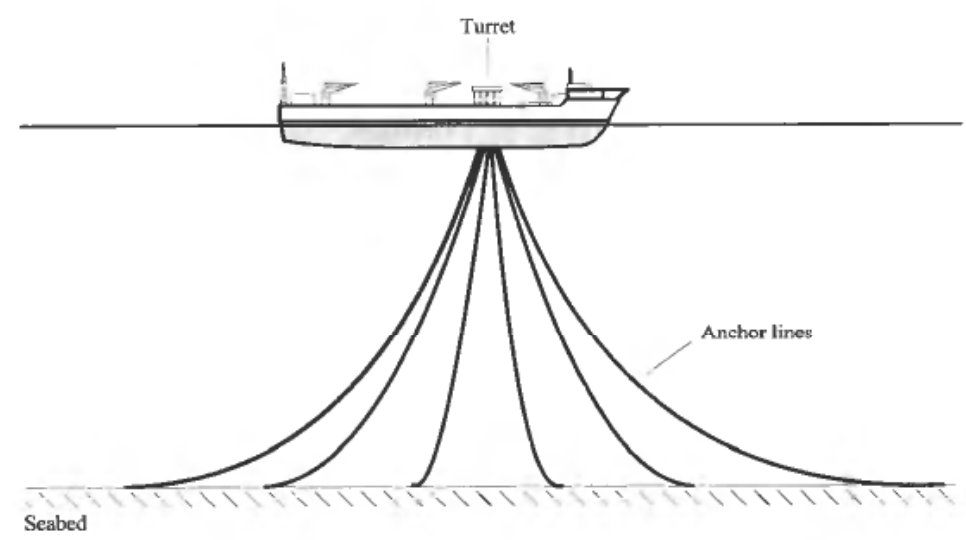

Figure 1. Moored vessel.

$\dagger$ Department of Engineering Cybernetics, Norwegian University of Science and Technology, N-7034 Trondheim, Norway, jann@itk.ntnu.no, tif@itk.ntnu.no

*ABB Industri AS, P.O. Box 6540 Rodeløkka, 0501 Oslo, Norway, asgeir.sorensen@ noina.abb.no 
ships, where station keeping is left entirely to the thruster system, see Fossen (1994) for an overview. This difference is also reflected in the number and the capacity of the thrusters installed. For turret-anchored vessels, the mooring system will normally provide only a small portion of the moment needed to keep the ship at the desired heading. Moreover, the positioning capability of the moored ship is strongly dependent on its ability to keep the heading such that the influence of the environmental loads is minimized. Thus, heading control by means of the thrusters is crucial for safe operation of such ships. Due to the physical properties of the mooring system, new control functionality and modes of operation are required compared to conventional DP operated ships. Slowly-varying excitation forces due to waves and wind may excite resonant oscillatory motions of the moored structure. Hence, an important function of the control system is to damp such motions by proper action of the thruster system. In rough weather situations a break in one anchor line may be critical for the station keeping ability and thereby the overall safety. A mooring line break will result in higher load in the remaining lines. The subsequent large transient motion of the vessel increases the risk of yet another break, and even more important, possible break in the risers. Hence, another important function in the control system is line break detection and line break compensation by the thrusters.

In this paper a mathematical model of a turret-anchored ship is presented. Focus is placed on the dynamic low-frequency model, where the effect of the restoring forces caused by the mooring system is treated. A control system for automatic thruster assisted position mooring (PM) systems is derived. This is an extension to the model-based DP control system design proposed in Sørensen et al. (1996).

\section{Mathematical Modelling}

\subsection{Kinematics}

Three different reference frames are used in position mooring, as illustrated in Fig. 2. The earth-fixed reference frame $X_{E} Y_{E} Z_{E}$ is located in the vicinity of the desired position of the vessel and defined as the field zero point (FZP). A vessel-parallel frame $X_{V} Y_{V} Z_{V}$ is fixed in the earth-fixed frame, rotated to the desired heading angle $\psi_{d}$, and then translated to the desired $x_{d}$ and $y_{d}$ position coordinates and represented by the vector

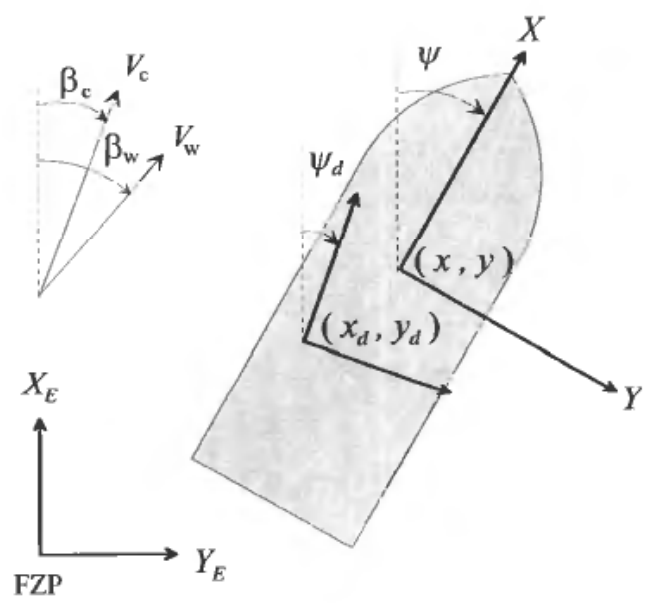

Figure 2. Reference frames. 
$\boldsymbol{\eta}_{d}=\left[x_{d}, y_{d}, \psi_{d}\right]^{T}$. The body-fixed $X Y Z$-frame is fixed to the vessel body with the origin located at the centre of turret (COT). It is assumed in the further modelling that the COT is located at the centre line of the vessel.

The linear velocities of the ship in the body-fixed frame and in the earth-fixed frame are related by the transformation:

$$
\dot{\boldsymbol{\eta}}=\mathbf{R}(\boldsymbol{\eta}) \boldsymbol{\nu},
$$

where the earth-fixed vessel position and heading, and the body-fixed surge, sway and yaw velocities are defined by the vectors $\boldsymbol{\eta}=[x, y, \psi]^{T}$ and $v=[u, v, r]^{T}$, respectively. The rotation matrix $\mathbf{R}(\boldsymbol{\eta})$ is:

$$
\mathbf{R}(\boldsymbol{\eta})=\mathbf{R}(\psi)=\left[\begin{array}{ccc}
\cos \psi & -\sin \psi & 0 \\
\sin \psi & \cos \psi & 0 \\
0 & 0 & 1
\end{array}\right] .
$$

Note that $\mathbf{R}^{-1}(\psi)=\mathbf{R}^{T}(\psi)$. Deviations from the desired position and heading in the vessel-parallel frame are given by:

$$
\mathbf{e}_{\eta}=\mathbf{R}^{T}\left(\boldsymbol{\eta}_{d}\right)\left(\boldsymbol{\eta}-\boldsymbol{\eta}_{d}\right)
$$

\subsection{Vessel Model}

The mathematical model describing the vessel dynamics is separated into a low-frequency (LF) model and a wave-frequency (WF) model. The WF motions are assumed to be caused by first-order wave loads. Although the vessel is attached to a mooring system, it is common to assume the WF motions to be little influenced by the mooring system. Assuming small amplitudes the WF motions can be represented by a linear model. The LF motions are assumed to be caused by second-order mean and slowly varying wave loads, wind loads, the mooring system and the thruster forces.

\subsubsection{Nonlinear Low-Frequency Model}

The low-frequency model of the moored vessel in the horizontal plane, decomposed in surge, sway and yaw motions, can be written as:

$$
\mathbf{M} \dot{\boldsymbol{\nu}}+\mathbf{C}_{\mathrm{RB}}(\boldsymbol{\nu}) \boldsymbol{\nu}+\mathbf{C}_{\mathrm{A}}\left(\boldsymbol{\nu}_{r}\right) \boldsymbol{\nu}_{r}+\mathbf{D}_{\mathrm{L}} \boldsymbol{\nu}+\mathbf{D}_{\mathrm{NL}}\left(\boldsymbol{\nu}_{r}, \boldsymbol{\gamma}_{r}\right) \boldsymbol{\nu}_{r}=\boldsymbol{\tau}_{\mathrm{wi}}+\boldsymbol{\tau}_{\mathrm{mo}}+\boldsymbol{\tau}_{\mathrm{wa} 2}+\boldsymbol{\tau}_{\mathrm{thr}},
$$

where the right hand expressions represent generalized external forces. ${ }^{1}$ Here, $\boldsymbol{\tau}_{\mathrm{thr}}$ is a vector of generalized control forces provided by the thruster system, $\boldsymbol{\tau}_{\mathrm{wi}}$ is the mean wind load vector, $\boldsymbol{\tau}_{\mathrm{wa} 2}$ the second-order wave drift load vector and $\boldsymbol{\tau}_{\mathrm{mo}}$ is a vector of generalized mooring forces. Modelling of the mooring system is treated in the next section.

$\mathbf{M}$ is the low-frequency mass matrix including hydrodynamic added mass, $\mathbf{C}_{\mathrm{RB}}(\boldsymbol{\nu})$ and $\mathbf{C}_{\mathrm{A}}\left(\boldsymbol{\nu}_{\tau}\right)$ are skew-symmetric Coriolis and centripetal matrices of the rigid-body and the added mass, respectively, $\mathbf{D}_{\mathrm{L}}$ is a strictly positive damping matrix, caused by linear wave drift damping and laminar skin friction and $\mathbf{D}_{\mathrm{NL}}\left(\boldsymbol{\nu}_{r}, \gamma_{r}\right)$ is a nonlinear damping matrix. In the latter, the effect of current is modelled, where $\boldsymbol{\nu}_{r}$ is the relative velocity vector with respect to water current and $\gamma_{r}$ is the relative drag angle. It is assumed that surge is decoupled from sway and yaw motion at low speed, which is reflected in the

\footnotetext{
'Throughout this paper forces in surge and sway and moment in yaw will be referred to as generalized forces or simply forces.
} 
structures of $\mathbf{M}$ and $\mathbf{D}_{\mathrm{L}}$. For details regarding this model, the reader is referred to Sørensen et al. (1996). In the DP literature the LF ship model is often given relative to centre of gravity. Thus, when using COT as origin for the body-fixed frame, an appropriate geometric transformation of (4) is needed.

\subsubsection{WF Model}

The vessel's wave frequency (WF) motions in surge, sway and yaw, represented by the vector $\boldsymbol{\eta}_{w}=\left[x_{w}, y_{w}, \psi_{w}\right]^{T}$, are mainly generated by the 1 st-order wave forces acting on the ship. This WF motion can be found by transfer functions in each degree of freedom, based on experimental data or by specialized software. However, in control design it is common to use linear approximations, represented by a state space model:

$$
\begin{aligned}
\dot{\boldsymbol{\xi}} & =\boldsymbol{\Omega} \boldsymbol{\xi}+\Sigma \mathbf{w}_{\mathrm{w}} \\
\boldsymbol{\eta}_{w} & =\Gamma \boldsymbol{\xi},
\end{aligned}
$$

where $\xi \in \mathfrak{R}^{p \cdot 3}, \mathbf{w}_{\mathrm{w}} \in \mathfrak{R}^{3}$ is a stochastic white noise vector and $p$ is the order of the linear WF motion model. In each degree of freedom, $i=1,2,3$, this can for example be a 2nd-order oscillator $(p=2)$ :

$$
\eta_{w i}(s)=\frac{\sigma_{i} s}{s^{2}+2 \zeta \omega_{o i} s+\omega_{o i}^{2}} w_{w i},
$$

as proposed in Sælid et al. (1983). Higher order models have also been proposed. In (5) $\omega_{o i}$ is the natural frequency, related to the dominating wave frequency in a wave spectrum, $\sigma_{i}$ is a scaling parameter related to the wave spectrum intensity and $\zeta$ is the relative damping ratio. From a practical point of view $\omega_{o i}$ will be a slowly varying parameter, depending on the sea-state, and has to be accordingly adapted by the control system.

\subsection{Mooring System}

Generally, a spread mooring system consists of $n$ pre-tensioned anchor lines connected to a turret of the ship, as illustrated in Fig. 1. The number of anchor lines may vary, typically from 6 to 12 lines. The location of the turret is decisive to the weather-vaning abilities of the moored vessel (Aalbers et al. 1995). When the turret is rotatable, the relative angle between the turret and the body-fixed frame is given by the turret angle, $\alpha_{t}$. In some systems the ship can rotate freely in yaw relative to the turret and in other systems the turret is locked under operation, but can be rotated manually by motor power. The length of each anchor line is adjusted by winches and determines the pre-tension and thus the stiffness of the mooring system. In this paper it is assumed that line length is manually controlled, and fixed around a working point. A typical mooring pattern with the turret angle indicated is shown in Fig. 3.

The anchor lines enter the turret through fairleads below the hull and the coordinates are defined as terminal points (TP). The anchor lines are composed of chain, wirelines or synthetic material, often partitioned into several segments of different types and properties.

\subsubsection{Single Line Modelling}

A profile of an anchor line is sketched in Fig. 4. The tangential force at a TP, often called the line tension, is denoted $T$, and its horizontal force component is denoted $H$. 


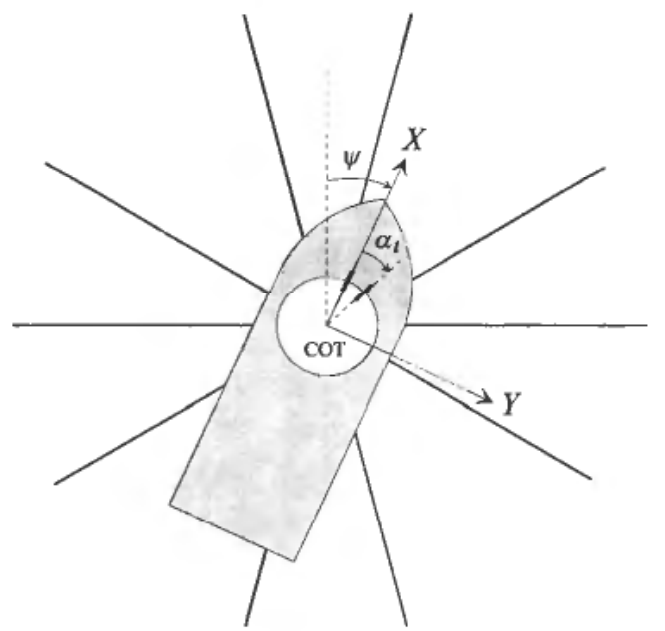

Figure 3. Definition of turret angle and a typical mooring pattern.

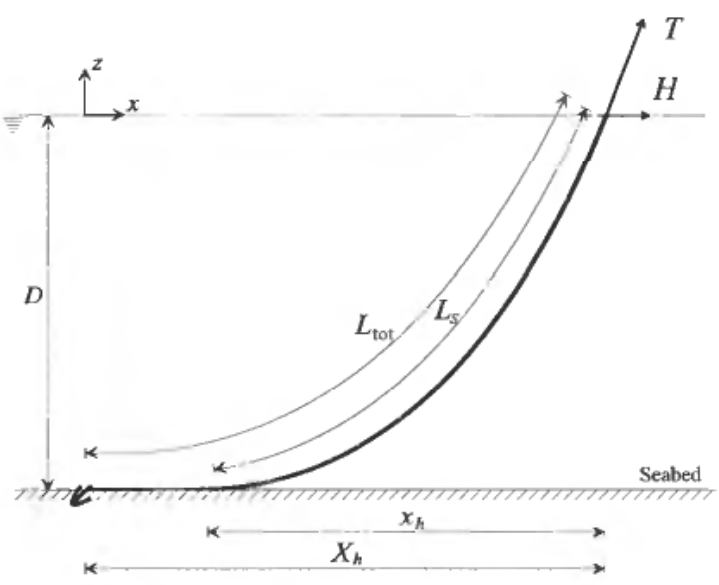

Figure 4. Line profile.

Moreover, $L_{s}$ is the suspended length, defined as the static cable length from the TP on the ship to the point where the line first touches the seabed. The latter point is called the touchdown point. $L_{l o t}$ is the total length of the cable, $D$ is the water depth, $x_{h}$ is the horizontal distance between the TP and the touchdown point, and $X_{h}$ is the horizontal distance between the TP and the anchor.

Mooring lines are subjected to three types of excitation (Triantafyllou 1994): Large amplitude LF motions, medium amplitude WF motions and small amplitude, very high frequency vortex-induced vibrations. Since these effects occur at different frequencies, the excitations can be treated separately in order to make the modelling tractable. In the control system design the focus will be placed on the mooring lines' influence on the LF model (4).

Let $T$ and $H$ be modelled as a combination of the static terms $\bar{T}$ and $\bar{H}$ and the dynamic terms $\tilde{T}$ and $\tilde{H}$ : 
$\overline{\mathrm{T}}$ (solid) and $\overline{\mathrm{H}}$ (dashed) at TP [kN]

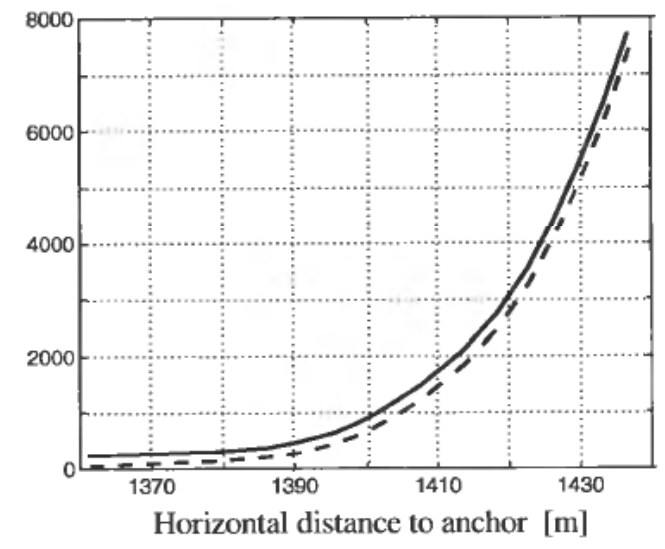

Figure 5. Line characteristics $(D=125 \mathrm{~m})$ : Static tension $\bar{T}$ (solid) and its horizontal component $\bar{H}$ (dashed) at TP as function of horizontal distance to anchor.

$$
T=\bar{T}+\tilde{T}, H=\bar{H}+\tilde{H} .
$$

When knowing the position of the line's upper end (TP) and the anchor position, it would be desirable to find the static tension and its horizontal component at the TP. This problem cannot be solved analytically. However, such distance/force relationships, often denoted the line characteristics, can be found using specialized software (Marintek 1995):

$$
\bar{T}=f_{T}\left(X_{h}\right), \bar{H}=f_{H}\left(X_{h}\right) .
$$

The line characteristics are found numerically by solving the catenary equations, where the influence of hydrostatic effects and the current profile along the line are taken into account. Typical line characteristics are plotted in Fig. 5.

By assuming horizontal waterbed, one-segment line with no bending stiffness and no elasticity, some simple, yet useful, relationships can be established (Faltinsen 1990):

$$
\begin{aligned}
\bar{T} & =\sqrt{\bar{H}^{2}+\left(w_{o} L_{s}\right)^{2}} \\
\bar{T} & =\bar{H}+w_{o} D \\
L_{s} & =\sqrt{D\left(D+\frac{2 \bar{H}}{w_{o}}\right)} \\
x_{h} & =\frac{\bar{H}}{w_{o}} \sinh ^{-1}\left(\frac{w_{o} L_{s}}{\bar{H}}\right) \\
x_{h} & =\frac{\bar{H}}{w_{o}} \cosh ^{-1}\left(\frac{w_{o} D}{\bar{H}}+1\right),
\end{aligned}
$$

where $w_{o}$ denotes the net weight per unit length of the line in water. For dynamic modelling of mooring lines and moored structures, the reader is referred to Triantafyllou (1994). 


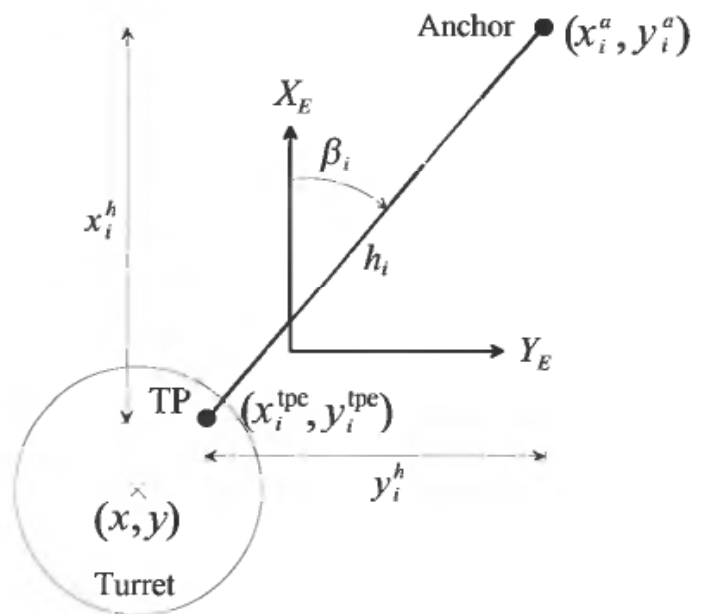

Figure 6. One anchor line in a mooring system.

\subsubsection{Spread Mooring System}

Let the horizontal force at the terminal point (TP) of each line be denoted $H_{i}, i=1 \ldots n$. Moreover, let $\left(x_{i}^{a}, y_{i}^{a}\right)$ denote the earth-fixed anchor position of line $i$ and $\left(x_{i}^{\text {pe }}, y_{i}^{\text {tpe }}\right)$ the earth-fixed positions of the line's corresponding TP on the turret. The latter coordinates will depend on the vessel position, heading and turret angle. If $\left(x_{i}^{\mathrm{t} 0}, y_{i}^{\mathrm{tp} 0}\right)$ denotes the terminal point coordinates relative to the body fixed frame (Fig. 6) at initial turret angle, $\alpha_{t}=\psi=0$, one can write:

$$
\begin{aligned}
& x_{i}^{\mathrm{tpe}}=x-\cos \left(\psi+\alpha_{t}\right) x_{i}^{\mathrm{tp} 0}+\sin \left(\psi+\alpha_{t}\right) y_{i}^{\mathrm{tp} 0} \\
& y_{i}^{\mathrm{tpe}}=y-\sin \left(\psi+\alpha_{t}\right) x_{i}^{\mathrm{tp} 0}-\cos \left(\psi+\alpha_{t}\right) y_{i}^{\mathrm{tp} 0}
\end{aligned}
$$

A model for the generalized mooring forces is formulated:

$$
\boldsymbol{\tau}_{\mathrm{mo}}=-\mathbf{R}^{T}\left(\psi+\psi_{w}\right) \mathbf{g}_{\mathrm{mo}}\left(\boldsymbol{\eta}, \boldsymbol{\eta}_{w} ; \boldsymbol{\nu}, \boldsymbol{\omega}, \alpha_{t}\right)-\mathbf{d}_{\mathrm{mo}}\left(\boldsymbol{\nu} ; \boldsymbol{\eta}, \boldsymbol{\eta}_{w}, \boldsymbol{\omega}, \alpha_{t}\right),
$$

which consists of a restoring term, $\mathbf{g}_{\mathrm{mo}}$, and an additional damping term, $\mathbf{d}_{\mathrm{mo}}$, due to the mooring system. Here, $\boldsymbol{\omega}$ represents dynamic states in the mooring lines. In some cases the effect of the heave, pitch and roll motion of the ship should also be taken into account. The earth-fixed restoring term is given as:

$$
\mathbf{g}_{\mathrm{mo}}=\sum_{i=1}^{n}\left[\begin{array}{c}
H_{i} \cos \beta_{i} \\
H_{i} \cos \beta_{i} \\
H_{i} \bar{x}_{i} \sin \beta_{i}-H_{i} \bar{y}_{i} \cos \beta_{i}
\end{array}\right],
$$

which simply is vectorial addition of the force contribution from each line where

$$
\begin{aligned}
\beta_{i} & =\operatorname{atan} 2\left(y_{i}^{h}, x_{i}^{h}\right) \\
x_{i}^{h} & =x_{i}^{a}-x_{i}^{\mathrm{tpe}}, y_{i}^{h}=y_{i}^{a}-y_{i}^{\mathrm{tpe}} \\
\bar{x}_{i} & =x-x_{i}^{\mathrm{ppe}}, \bar{y}_{i}=y-y_{i}^{\mathrm{tpe}}
\end{aligned}
$$

and $\beta_{i}$ is the earth-fixed direction of line $i$ as illustrated in Fig. 6. In cases where the ship can rotate freely relative to the turret, $\alpha_{t}=-\psi$, there will be no restoring moment 
in yaw on the ship from the mooring system. The horizontal distance from the anchor to the TP of each line $i$ is:

$$
h_{i}=\sqrt{\left(x_{i}^{h}\right)^{2}+\left(y_{i}^{h}\right)^{2}} .
$$

In a quasi-static approach the restoring forces $\mathbf{g}_{\mathrm{mo}}$ are treated as function of the low frequency ship position and heading $\boldsymbol{\eta}$ only:

$$
\mathbf{g}_{\mathrm{mo}} \stackrel{q . s}{\approx} \overline{\mathbf{g}}_{\mathrm{mo}}\left(\boldsymbol{\eta} ; \alpha_{t}\right)
$$

where the horizontal force contributions $H_{i}$ in (11) are replaced by the static line characteristics for each line $i$ by:

$$
\bar{H}_{i}=f_{H i}\left(h_{i}\right) \text {, }
$$

which is a function of the horizontal distance $h_{i}$ between TP and the anchor of each line. It is common to let the earth-fixed frame be fixed in the natural equilibrium point of the mooring system, such that $\overline{\mathbf{g}}_{\mathrm{mo}}(\mathbf{0})=\mathbf{0}$ when no external loads are acting on the ship. The LF modelling of the mooring forces becomes quasi-static if disregarding the dynamic effects in the mooring lines and using (13) in the LF ship model (4). According to Triantafyllou (1994) it can be assumed that the mooring system does not affect the Ist-order wave-induced motion of the vessel. One the contrary, however, superimposing the WF vessel motions with the LF motions yields a significant increase in the LF damping, due to the transverse response of the lines along the catenary profile. These slowly-varying mooring damping forces vary in a non-linear manner with the wave amplitude and the frequency. For typical wave spectra the effective drag coefficients increase in a magnitude of 2 to 4, see Huse and Matsumoto (1989). Similar increased damping effects are also reported in Triantafyllou and Yue (1994).

\subsubsection{Linear Mooring Model}

As shown in Faltinsen (1990) a mooring line will have a spring effect on a body attached at the line's upper end. Around a working point the line characteristics (14) can be linearized by:

$$
\bar{H}_{i}=\bar{H}_{o i}+c_{i} \Delta h_{i},
$$

where $\bar{H}_{o i}$ is the average horizontal force in the working point $h_{i o}$ and

$$
c_{i}=\left.\frac{d f_{H i}}{d h_{i}}\right|_{h_{i}=h_{i o}}
$$

is the slope of the line characteristics (14) at $h_{i o}$. Assuming fixed anchor line length and neglecting the influence of the current field along the line profile, the generalized mooring forces (10) in a working point $\boldsymbol{\eta}_{o}=\left[x_{o}, y_{o}, \psi_{o}\right]^{T}$ can be approximated by:

$$
\boldsymbol{\tau}_{\mathrm{mo}}\left(\boldsymbol{\eta}, \boldsymbol{\nu} ; \alpha_{t}\right) \simeq-\mathbf{D}_{v} \boldsymbol{\nu}-\mathbf{G}_{e} \mathbf{e}-\mathbf{R}^{T}\left(\psi_{o}\right) \overline{\mathbf{g}}_{\mathrm{mo}}\left(\boldsymbol{\eta}_{o} ; \alpha_{t}\right),
$$

where $\mathbf{e}=\mathbf{R}^{T}\left(\psi_{o}\right)\left(\boldsymbol{\eta}-\boldsymbol{\eta}_{o}\right)$ represents deviations from the linearization point $\boldsymbol{\eta}_{o}$, and

$$
\begin{aligned}
\mathbf{G}_{e} & \left.\triangleq \frac{\partial}{\partial \mathbf{e}}\left(\mathbf{R}^{T}(\psi) \overline{\mathbf{g}}_{\mathrm{mo}}\left(\boldsymbol{\eta} ; \alpha_{t}\right)\right)\right|_{\mathbf{e}=\mathbf{0}} \\
& =\mathbf{R}^{T}\left(\psi_{o}\right) \mathbf{G}_{\eta} \mathbf{R}\left(\psi_{o}\right)-\mathbf{T}\left(\psi_{o}\right) \overline{\mathbf{g}}_{\mathrm{mo}}\left(\boldsymbol{\eta}_{o} ; \alpha_{t}\right)[0,0,1] \\
\mathbf{D}_{v} & \left.\triangleq \frac{\partial}{\partial \boldsymbol{\nu}}\left(\mathbf{d}_{\mathrm{mo}}\left(\boldsymbol{\nu} ; \boldsymbol{\eta}, \boldsymbol{\eta}_{w}, \boldsymbol{\omega}, \alpha_{t}\right)\right)\right|_{\boldsymbol{\nu}=\mathbf{0}}
\end{aligned}
$$


where

$$
\begin{gathered}
\mathbf{T}\left(\psi_{o}\right)=\left[\begin{array}{ccc}
\sin \psi_{o} & -\cos \psi_{o} & 0 \\
\cos \psi_{o} & \sin \psi_{o} & 0 \\
0 & 0 & 0
\end{array}\right], \\
\mathbf{G}_{\eta}=\left.\frac{\partial \overline{\mathbf{g}}_{\mathrm{mo}}}{\partial \eta}\right|_{\eta=\eta_{o}}=\left\{g_{i j}^{\eta}\right\}, \\
g_{11}^{\eta}=\sum_{i=1}^{n} c_{i} \cos ^{2} \beta_{i o}, \quad g_{12}^{\eta}=g_{21}^{\eta}=\sum_{i=1}^{n} c_{i} \cos \beta_{i o} \sin \beta_{i o}, \\
g_{22}^{\eta}=\sum_{i=1}^{n} c_{i} \sin ^{2} \beta_{i o}, \quad g_{13}^{\eta}=g_{31}^{\eta}=\sum_{i=1}^{n} c_{i} \bar{N}_{i o} \cos \beta_{i o}, \\
g_{33}^{\eta}=\sum_{i=1}^{n} c_{i} \bar{N}_{i o}^{2}, \quad g_{23}^{\eta}=g_{32}^{\eta}=\sum_{i=1}^{n} c_{i} \bar{N}_{i o} \sin \beta_{i o},
\end{gathered}
$$

and $\bar{N}_{i o}=\bar{x}_{i o} \sin \beta_{i o}-\bar{y}_{i o} \cos \beta_{i o}$. Subscript $o$ denotes value evaluated at the linearization point. The additional linear damping matrix $\mathbf{D}_{v}$ due to the mooring system is assumed to have the same structure as the linear damping matrix $\mathbf{D}_{L}$ in (4), i.e. surge is decoupled from sway and yaw. In cases where the ship can rotate freely relative to the turret there will be no restoring moment in yaw on the ship from the mooring system such that $g_{i 3}^{\eta}=g_{3 i}^{\eta} \equiv 0, i=1,2,3$.

When knowing the LF vessel mass matrix including added mass $\mathbf{M}$ and the static line characteristics (14) for all $n$ lines, it is a priori possible to predict the resonance periods of the moored vessel by using the linearized mooring model (17). In the following it is assumed that the ship moves in surge only, in the direction of the linearization angle $\psi_{o}$. Let $e_{1}$ denote the deviation from the linearization point $\boldsymbol{\eta}_{o}$ in this direction. Hence, $u=\frac{d e_{1}}{d t}$ and the surge equation of motion can be written:

$$
\left(m-X_{\dot{u}}\right) \dot{u}+d(u) u+g_{11}^{e} e_{1}=\tau_{x}(t),
$$

where $d(u) u$ is a nonlinear damping term, $m$ is the vessel mass, $X_{\dot{u}}$ is added mass in surge, $g_{11}^{e}$ is the upper left matrix element of $\mathbf{G}_{e}(18)$ and $\tau_{x}(t)$ a dynamic excitation force. From (20) the undamped resonance period in surge can be approximated by:

$$
T_{o}=\frac{2 \pi}{\omega_{o}}=2 \pi \sqrt{\frac{m-X_{\dot{u}}}{g_{11}^{e}}} .
$$

The resonance period in sway can be found analogously. In a practical case resonance oscillations of a moored vessel will be excited by slowly-varying forces due to wind and waves. According to Triantafyllou (1994) a typical resonance period for moored vessels are 100 seconds or larger, depending on water depth and pretension. The motions may be in the magnitude of $5 \%$ of the water depth.

\section{Control System}

In this chapter the controller functionality, modes of operation and control system design is addressed. The PM controller design is an extension of the conventional DP design, see Sørensen et al. (1996) for details. The feedback controller is designed using LQG theory. In most cases accurate measurements of the ship velocities, $v$, are not available and thus must be computed by a state observer. Moreover, the wave frequency motion of the ship should not be counteracted by the propulsion system, which would result in intolerable wear and tear of the thruster devices. So-called wave-filtering 
techniques, which separates the position and heading measurements into low-frequency and wave-frequency components, are used in the observer design. As in the DP case, feedback is produced from low-frequency position and heading estimates and corresponding estimated velocities. In the observer design, Kalman filter theory is utilized in combination with adaptive gain-scheduling techniques.

\subsection{Control Modes of Operation}

The surge, sway and yaw degrees of freedom (DOF) can individually be controlled in the following modes of operation:

- Manual Control. With a joystick the operator can generate manual force setpoints in surge and sway and with a rotation knob generate a manual moment setpoint in yaw.

- Damping Control. Feedback is taken from estimated vessel velocities and the objective is to let the control system regulate the vessel speed in a DOF to zero.

- Setpoint Control. Feedback is produced from both estimated velocities and low-frequency position/yaw angle estimates. The objective is to automatically keep the DOF at the specified setpoint position or heading.

- Tracking Control. The vessel automatically tracks a smooth reference trajectory which is computed from the present setpoint to a new position or heading setpoint.

The combination of damping control in surge and sway and setpoint control of heading is often used, especially in bad weather. Effective damping will reduce possible large oscillatory motions and thus reduce the stress on the mooring system. Moreover, the vessel will tend to an equilibrium position where the mean environmental forces are balanced by the mooring forces. Assuming a proper heading setpoint, this equilibrium position will be optimal with respect to the thrust usage and the fuel consumption. For turret-anchored ships automatic heading control is the most important function of the control system. By keeping the heading against the weather the effect of the environmental loads, and thereby the stress on the mooring system, will be minimized.

When tracking control is performed in all $3 \mathrm{DOF}$ or in surge and sway, this is often defined as a Marked Position operation. Tracking control in heading only is called a Marked Heading operation.

\subsection{Line Break Detection and Compensation}

Line break in one or several lines are detected in order to have an immediate compensation by the thrusters. Irregular tension measurements triggers the line break detection algorithm. When a line break is detected, automatic feedforward thrust in surge, sway and yaw, $\tau_{\mathrm{l}}$, is computed. The purpose of this line break compensation term is to ease the load in the surrounding lines and thus avoiding yet another line break.

\subsection{Feedforward Control Law}

In addition to the line break compensation term, wind feedforward control can be applied. By measuring the wind velocity and direction estimates of the wind forces, $\hat{\tau}_{\text {wind }}$, can be found. Such wind feedforward can be enabled individually in each DOF by the operator. Normally wind feedforward is enabled in yaw DOF only, since the often large stationary wind load in surge and sway should be compensated by the mooring 
system. The wind force estimates are multiplied by a diagonal gain matrix, and the feedforward term is taken to be:

$$
\boldsymbol{\tau}_{\mathrm{wff}}=-\mathbf{G}_{w} \hat{\boldsymbol{\tau}}_{\mathrm{wind}} .
$$

In tracking control a smooth reference trajectory $\boldsymbol{\eta}_{d} \in C^{r}$ is generated. In order to improve the performance of the system during tracking operations, a model reference feedforward, $\boldsymbol{\tau}_{\mathrm{rff}}$, is computed according to:

$$
\boldsymbol{\tau}_{\mathrm{rff}}=\mathbf{M a}_{d}+\mathbf{D}_{L} \boldsymbol{\nu}_{d}+\mathbf{C}_{\mathrm{RB}}\left(\boldsymbol{\nu}_{d}\right) \boldsymbol{\nu}_{d}+\mathbf{C}_{\mathrm{A}}\left(\boldsymbol{\nu}_{d}\right) \boldsymbol{\nu}_{d}
$$

where $\mathbf{a}_{d}$ and $\boldsymbol{\nu}_{d}$ are the body-fixed reference acceleration and velocity vectors, respectively.

\subsection{Feedback Control Law}

The control deviation vector is defined as:

$$
\hat{\mathbf{e}}=\left[\begin{array}{lll}
\hat{\mathbf{e}}_{v}^{T} & \hat{\mathbf{e}}_{\eta}^{T} & \hat{\mathbf{e}}_{t}^{T}
\end{array}\right]^{T},
$$

where $\hat{\mathbf{e}}_{v}$ is a vector of LF velocity estimates, $\hat{\mathbf{e}}_{\eta}$ is a vector of LF position and yaw angle estimates relative to the vessel-parallel frame and $\hat{\mathbf{e}}_{t}$ is a thrust deviation vector:

$$
\begin{aligned}
& \hat{\mathbf{e}}_{v}=\left[\begin{array}{lll}
\hat{u} & \hat{v} & \hat{r}
\end{array}\right]^{T} \\
& \hat{\mathbf{e}}_{\eta}=\mathbf{R}^{T}\left(\psi_{d}\right)\left[\hat{x}-x_{d}, \hat{y}-y_{d}, \hat{\psi}-\psi_{d}\right]^{T} \\
& \hat{\mathbf{e}}_{t}=\hat{\boldsymbol{\tau}}_{\mathrm{thr}}-\boldsymbol{\tau}_{c},
\end{aligned}
$$

where $\hat{\boldsymbol{\tau}}_{\mathrm{thr}}$ is the estimated thruster force vector, based on azimuth angle measurements and estimates of the thruster force developed by each thruster device. The total commanded thrust in surge, sway and yaw is denoted $\tau_{\mathrm{c}}$. In the state estimation, Kalman filter theory is utilized. Based on a linear vessel model, the linear quadratic controller (LQ) is derived. The LQ term of the commanded thrust is partitioned into:

$$
\begin{aligned}
\boldsymbol{\tau}_{\mathrm{LQ}} & =\boldsymbol{\tau}_{v}+\boldsymbol{\tau}_{\eta}+\boldsymbol{\tau}_{t}=-\mathbf{G}_{\mathrm{LQ}} \hat{\mathbf{e}} \\
& =-\mathbf{G}_{v} \hat{\mathbf{e}}_{v}-\mathbf{G}_{\eta} \hat{\mathbf{e}}_{\eta}-\mathbf{G}_{t} \hat{\mathbf{e}}_{t} .
\end{aligned}
$$

Integral action, $\tau_{i}$, is added to the linear quadratic terms in order to avoid steady-state offsets from desired position and heading.

\subsection{Resulting Controller}

As discussed earlier, it is possible to change between different control modes of operation. The total commanded thrust in surge, sway and yaw is written:

$$
\boldsymbol{\tau}_{c}=\overline{\mathbf{S}}_{m}\left(\boldsymbol{\tau}_{v}+\mathbf{S}_{\eta} \boldsymbol{\tau}_{\eta}+\boldsymbol{\tau}_{t}+\mathbf{S}_{\eta} \boldsymbol{\tau}_{i}+\mathbf{S}_{w} \boldsymbol{\tau}_{\mathrm{wff}}+\mathbf{S}_{\eta} \boldsymbol{\tau}_{\mathrm{rff}}+\mathbf{S}_{\eta} \boldsymbol{\tau}_{\mathrm{b}}\right)+\mathbf{S}_{m} \boldsymbol{\tau}_{m},
$$

where the $\mathbf{S}$ matrices are control system dependent diagonal matrices of zeros or ones along the diagonal. When operating with setpoint control or tracking control in a DOF the corresponding diagonal element in $\mathbf{S}_{\eta}$ will be equal to 1 and 0 otherwise. $\overline{\mathbf{S}}_{m}$ denotes the logical inverse to the diagonal of $\mathbf{S}_{m}$, such that no automatic control of a DOF is possible when the system is operating in manual control in the corresponding DOF. Wind feedforward can be enabled in each DOF by the operator, which is reflected in the $\mathbf{S}_{w}$ matrix.

Since the controller design is performed for the 3 degrees of freedom model (4), a commanded thrust $\boldsymbol{\tau}_{c}$ in generalized forces will be computed by the controller. The problem of finding the corresponding control inputs (force and direction setpoint) of 


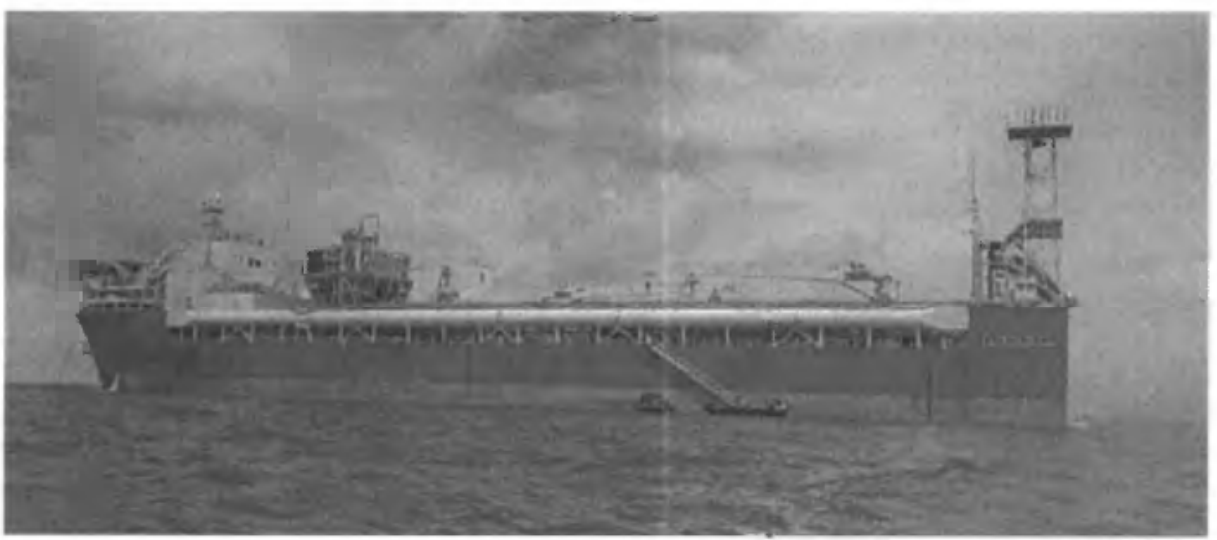

Figure 7. Balder FPU.

each thruster device is called thrust allocation and is thoroughly treated in Sørdalen (1996). The propeller and thruster devices can be controllable pitch propeller (CPP) with fixed speed, controllable speed with fixed pitch propeller (FPP), or controllable pitch and speed in combination. Conventionally, the setpoint of each thruster is not a force setpoint, but rather desired pitch or speed. The setpoints are determined from stationary propeller force to speed/pitch relations based on information about thruster characteristics and bollard pull tests provided by the thruster manufacturer. In such schemes, the unpredictable load variations will not be accounted for. In Sørensen et al. (1997) a new method based on torque control and power control of the propeller and thruster devices is introduced by using propeller forces to torque and power mappings.

\section{Case Study}

The control system described in this paper has been implemented as a part of the ABB Integrated Thrust Control System (ITCS), see Fig. 11 for a typical configuration overview. This system was installed on the Balder FPU, a FPSO contracted by Esso, see Fig. 7.

The Balder FPU is equipped with 3 azimuth thrusters, one at the fore and two at the aft, each designed to develop a maximum thrust of approximately $540 \mathrm{kN}$. The ship length between perpendiculars is approximately $200 \mathrm{~m}$ and the dead weight is approximately 73,000 tons. The mooring system considered consists of 10 anchor lines and the water depth at the field is $125 \mathrm{~m}$.

Computer simulations are performed with significant wave height $6.8 \mathrm{~m}$, wave peak period $10.5 \mathrm{sec}$., current velocity $0.45 \mathrm{~m} / \mathrm{s}$ and wind velocity $20 \mathrm{~m} / \mathrm{s}$. The earth-fixed wind direction is $205 \mathrm{deg}$, the current direrction $205 \mathrm{deg}$ and the mean wave direction $175 \mathrm{deg}$. Initially the control system is operating with only heading setpoint control. After 500 seconds damping control is selected in surge. Sway is manually controlled by the joystick with zero force setpoint in this case. After 1000 seconds a Marked Heading operation is performed, with new desired heading of 10 degrees. Earth-fixed vessel position and heading relative to FZP are presented in Fig. 8. The corresponding surge velocity is presented in Fig. 9, and in Fig. 10 the line tension in the most loaded line is presented. In this rough weather situation the heading control works satisfactorily, also when performing a Marked Heading operation. The effect of damping in surge is evident, since the dynamic surge motion and the line tensions are reduced. 

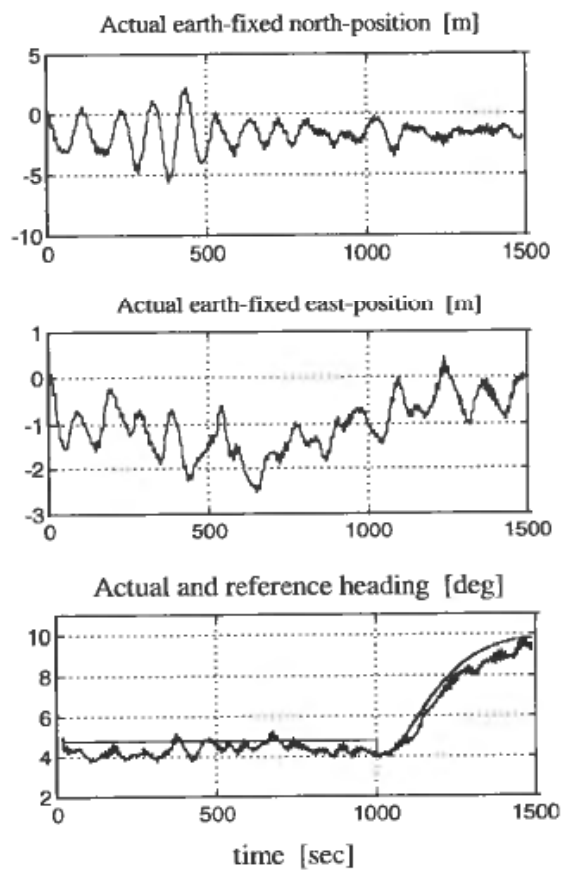

Figure 8. Earth-fixed position and heading relative to FZP.

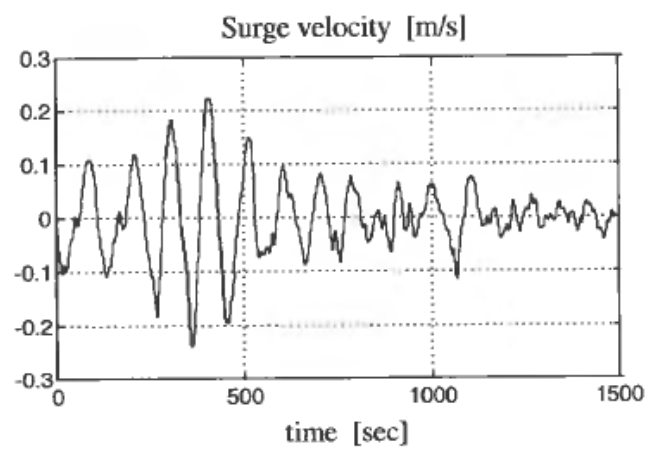

Figure 9. Surge velocity.

\section{Conclusions}

A mathematical model of a turret-moored ship was derived. A control system for automatic thruster assisted position mooring was proposed, using model-based controller design. The design was an extension to existing DP control systems, where the effect of the mooring system was taken into account. The control system functionality was described, along with the new control functions needed in position mooring (PM) systems. Automatic heading setpoint control has shown to be the most important function for turret-anchored ships. In bad weather damping control of surge and sway is necessary to reduce possible oscillatory motions induced by slowly varying environmental loads. Computer simulations in the time domain have been performed 


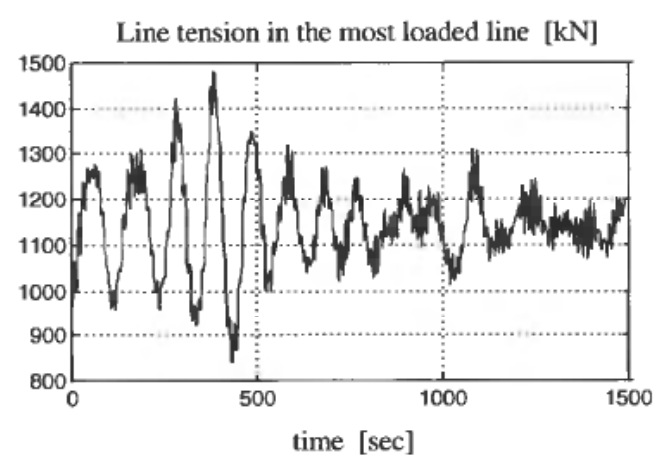

Figure 10. Line tension in the most loaded line.

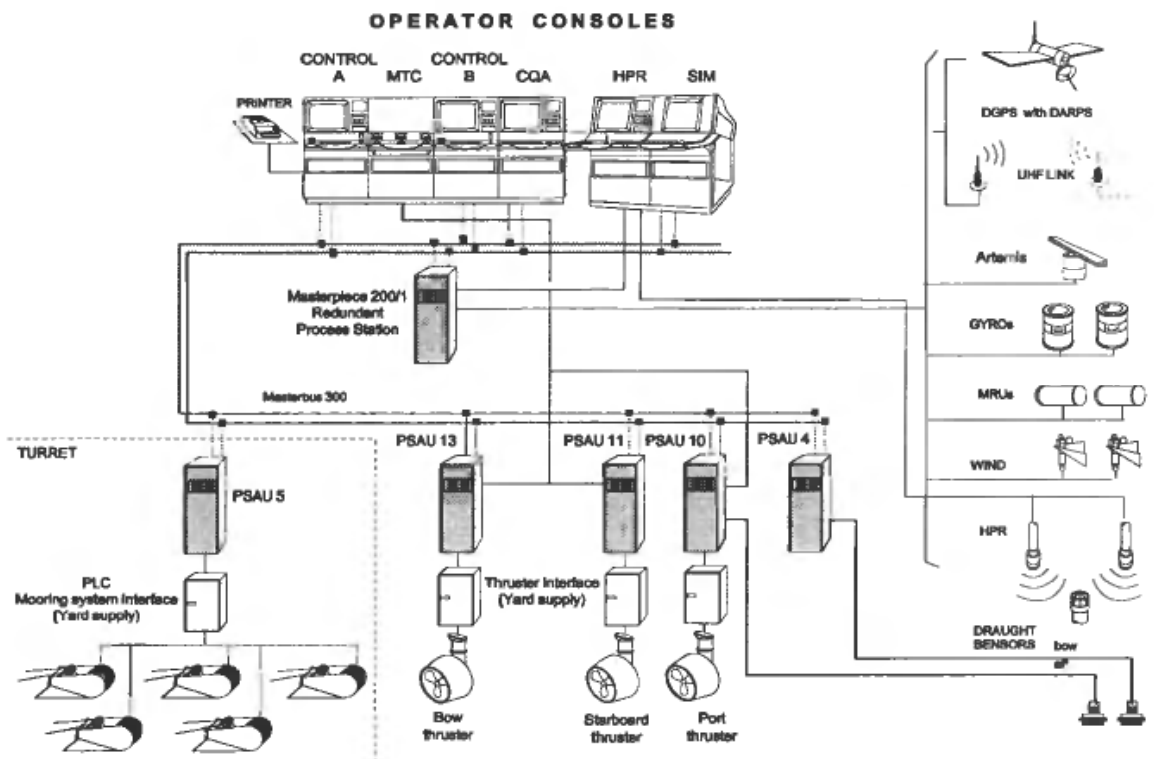

Figure 11. The ABB ITCS configuration for Balder FPU.

with the proposed control system. The performance of the system with automatic heading control and damping control in surge has been demonstrated by simulations.

\section{ACKNOWLEDGEMENTS}

This work is sponsored by The Research Council of Norway and ABB Industri AS and they are gratefully acknowledged.

REFERENCES

AAlbers, A. B., JANSE, A. W. and DE BOOM, W. C. (1995). DP assisted and Passive Mooring for FPSO's. 27th Annual Offshore Technology Conference, ppr. 7722, pp. 281-288, Houston, Texas.

Faltinsen. O. M. (1990). Sea Loads on Ships and Offshore Structures, Cambridge University Press.

Fossen, T. I. (1994). Guidance and Control of Ocean Vehicles, John Wiley and Sons Ltd. 
Huse, E. and Matsumoto, K. (1989). Mooring Line Damping Due to First- and Second-Order Vessel Motion. 21st Annual Offshore Technology Conference, Procs. Vol. 4, ppr. 6137, pp. 135-148, Houston, Texas.

MARINTEK (1995). MIMOSA User's Documentation. Marintek report No. 519616.00.02, Trondheim, Norway.

Selid, S., Jenssen, N. A. and Balchen, J. G. (1983). Design and Analysis of a Dynamic Positioning System Based on Kalman Filtering and Optimal Control. IEEE Trans. on Automatic Control, AC-28, pp. 331-339.

SøRDALEN, O. J. (1996). Thruster Allocation: Singularities and Filtering. IFAC World Congress, Vol. Q, pp. 369-374, San Francisco, CA.

Sørensen, A. J., Sagatun, S. I. and Fossen, T. I. (1996). Design of a Dynamic Positioning System using Model-Based Control, Journal of Control Engineering Practice, 4(3).

Sørensen, A. J., Ådnanes, A. K., Fossen, T. I. and Strand, J. P. (1997). A New Method of Thruster Control in Positioning of Ships Based on Power Control. Proc. 4th IFAC Conf. on Manoeuvring and Control of Marine Craft, pp. 172-179, Brijuni, Croatia.

Triantafyllou, M. S. (1994). Cable Mechanics for Moored Floating Systems. BOSS'94, pp. 67-77, Boston, MA.

Triantafyluou, M. S. and Yue, D. K. P. (1994). Damping of Moored Floating Structures. 26th Annual Offshore Technology Conference, Procs. Vol. 3, ppr. 7489, pp. 215-224, Houston, Texas. 